\title{
EUV spectroscopy of highly charged xenon ions
}

\author{
C. Biedermann*a , R. Radtke ${ }^{\mathrm{a}}$, G. Fußmann ${ }^{\mathrm{a}}$, J.L. Schwob ${ }^{\mathrm{b}}$, P. Mandelbaum ${ }^{\mathrm{b}}$ \\ ${ }^{a}$ Institut für Physik der Humboldt-Universität zu Berlin, Lehrstuhl Plasmaphysik, Newtonstraße 15, \\ D-12489 Berlin and Max-Planck-Institut für Plasmaphysik, EURATOM Association, Germany \\ ${ }^{\mathrm{b}}$ Racah Institute of Physics, The Hebrew University, 91904 Jerusalem, Israel
}

At the Berlin Electron Beam Ion Trap facility we investigated the radiation of highly charged xenon ions in the extreme ultraviolet wavelength range using a $2 \mathrm{~m}$ grazing incidence spectrometer. For $\mathrm{Rb}-$ like $\mathrm{Xe}^{17+}$ to $\mathrm{Cu}-\mathrm{like}$ $\mathrm{Xe}^{25+}$ ions 37 individual lines have been registered in the range between 90 and $240 \AA$ and identified by performing atomic structure calculations with the HULLAC code. The $4 s-4 p$ resonance lines of $\mathrm{Cu}$-like and $\mathrm{Zn}$-like $\mathrm{Xe}$ ions are found to be in good agreement with measurements at tokamaks and MCDF calculations. The experimental wavelengths for ions involving a larger number of electrons in the $n=4$ shell deviate slightly from the predicted values. Several lines observed with the BerlinEBIT had been previously seen but not identified at the tokamak and can now be classified.

\section{Introduction}

Xenon is envisaged as a coolant gas to be injected into the edge plasma of future large tokamaks, such as the International Thermonuclear Experimental Reactor (ITER), reducing the heat load on the plasma-facing components by radiative cooling [1]. Further, xenon admixtures in fusion plasmas serve as diagnostics for a variety of plasma properties including electron temperature and density, as well as ion temperature and impurity transport. Xe ions produced under hightemperature plasma conditions emit strongly in the extreme ultraviolet spectral range. In order to predict the effect of xenon injection on the performance of fusion plasmas and explore its diagnostic applications, accurate spectroscopic data are needed, which is so far only very sparsely available from experiment or theory. Furthermore, recent interest in applying the EUV radiation of xenon ions to lithography has led to the construction of light sources based on laserproduced plasmas or gas discharges [2]. In the effort to optimize for maximum radiation output detailed understanding of the origin of the radiation is necessary.

For $\mathrm{Cu}$-like ions the spectrum is dominated by

*Corresponding author: Fax: +49-30-2093-7549. E-mail address: biedermann@ipp.mpg.de prominent lines originating from the excitation of a single valence electron outside a closed $3 d^{10}$ core. The $4 s-4 p$ resonance transitions have been observed and calculated for many ions along the isoelectronic sequence and provide an important benchmark for precision and atomic structure theory [3]. In a plasma similar to that of a tokamak a range of charge states is present simultaneously, and for highly charged Xe ions several lines corresponding to $4 s-4 p$ transitions are in the EUV spectral range. Previous investigations at TFR tokamak measured a plenitude of Xe lines, but due to the superposition of the emissions of many charge states only few transitions could be identified unambiguously [4]. Measurements at the W7-AS stellerator show a wealth of lines with similar problems to tag the ion charge state [5]. With an EBIT's capability to selectively produce a limited, narrow distribution of charge states confined for a long observation time we could measure the radiation of highly charged Kr-like $\mathrm{Xe}^{18+}$ to $\mathrm{Cu}$-like $\mathrm{Xe}^{25+}$ ions, successively opening the $4 p$ and $4 s$ subshells in the wavelength range between 90 and $270 \AA$. With the help of atomic structure calculations using the HULLAC suite of codes [6] and a collisional radiative model we could assign transitions producing the observed lines and clarify the identifications of the TFR and W7-AS measurements. 


\section{Experimental method}

Highly charged xenon ions are produced, excited and confined for extended observation times in the Berlin Electron Beam Ion Trap (EBIT) [7]. A strongly compressed monoenergetic electron beam (typical beam current $10 \mathrm{~mA}$ resulting in $n_{e} \sim 5 \cdot 10^{10} \mathrm{~cm}^{-3}$ ) serves (a) to create by successive impact ionization highly charged ions limited in charge state by the electron beam energy $E_{\text {beam }}$, (b) to radially trap ions by the space charge potential and (c) to excite the ions. Axial trapping is provided by a 3 -sectioned drift tube structure allowing for periodical emptying of the trap to prevent accumulation of high-Z background ions. Additional radial ion trapping is provided by the 3 Tesla axial magnetic field compressing the electron beam. The target species xenon is fed as neutral gas continuously into the trapping region where it is ionized by the electron beam.

The EUV radiation from the ions is analyzed with a high-resolution $2 \mathrm{~m}$ SchwobFraenkel grazing-incidence spectrometer specifically adapted to the EBIT geometry [8]. Photons emitted by the ions in the electron beam are focused with a 6 -m-curvature grazing-incidence mirror on to the entrance slit $(18 \mu \mathrm{m})$ of the spectrometer, which in this experiment is equipped with a $600 \ell / \mathrm{mm}$ grating, mounted at an incidence angle of $2^{\circ}$ with a blaze angle of $3.5^{\circ}$. Moving the detector assembly (a $\mathrm{MgF}_{2}$-coated microchannelplate stack followed by a phosphor screen image intensifier viewed with a thermoelectrically cooled charge-coupled device camera) with high precision along the $2 \mathrm{~m}$ Rowland circle, spectral information in the range 30 to $1000 \AA$ can be recorded. The wavelength calibration was performed using the Lyman series of HeII and wellknown lines in first and second order diffraction for C- to Li-like Neon [9].

CCD images are recorded by successively decreasing the electron beam energy in small increments of about $60 \mathrm{eV}$ in order to follow how the radiation of certain ions ceases as soon as the ionization threshold for the production of that stage is reached. The electron beam energy $E_{\text {beam }}$ is determined by the acceleration potential at the drift tube assembly, taking corrections for the space charge of the electron beam and the ion inventory into account. The uncertainty in $E_{\text {beam }}$ is $\pm 20 \mathrm{eV}$ originating mainly from the estimate of the number of trapped ions. $E_{\text {beam }}$ has a spread of about $30 \mathrm{eV}$ FWHM.

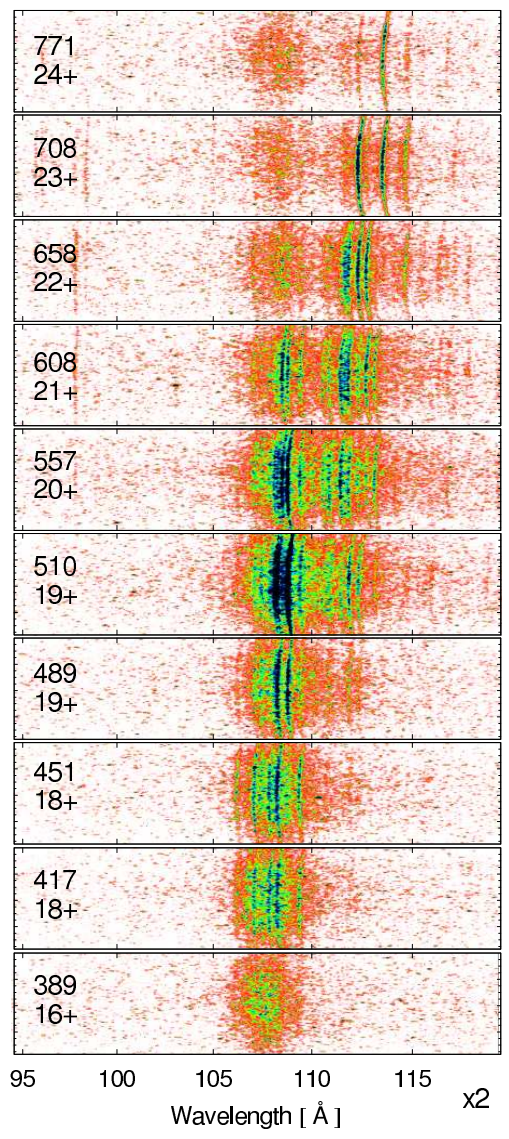

Figure 1. CCD images of spectral lines from highly charged Xe ions measured in second order diffraction. The labels mark the energy of the ionizing electron beam in $\mathrm{eV}$ and the highest allowed charge state of xenon corresponding to the given electron beam energy. 
Table 1

Experimental and theoretical wavelengths of $\mathrm{Cu}$-like and Zn-like Xe ions in $[\AA]$. The ground state of $\mathrm{Cu}$-like $\mathrm{Xe}^{25+}$ is $3 d^{10} 4 s^{2} S_{1 / 2}$ and of Zn-like $\mathrm{Xe}^{24+}$ is $4 s^{2}{ }^{1} S_{0}$. Ionization energies $\mathrm{E}_{i}$ are calculated with HULLAC. The experimental line intensity is estimated as vs (very strong), s (strong), m (moderate), w (weak) and vw (very weak). The theoretical line intensity is given in [ photons $/ \mathrm{s}$ ion ] for $n_{e}=10^{12} \mathrm{~cm}^{-3}$. The wavelength uncertainty for the measurements at the BerlinEBIT amounts to $0.058 \AA$, at TFR to $0.05 \AA$, at PLT to $0.5 \AA$ and at TEXT to $0.005 \AA$.

\begin{tabular}{lcllclcccc}
\hline Ion & \multicolumn{2}{c}{ BerlinEBIT } & Transition & \multicolumn{2}{c}{ HULLAC } & TFR [4] & PLT [10] & PLT [3] & TEXT [11] \\
& $\lambda$ & Int. & & & $\lambda$ & Int. & $\lambda$ & $\lambda$ & $\lambda$ \\
\hline $\mathrm{Xe}^{25+} \mathrm{Cu}-$ like & 173.877 & vs & $4 s{ }^{2} S_{1 / 2}-4 p{ }^{2} P_{3 / 2}$ & 174.09 & 3800 & 173.91 & 173.90 & 173.915 & 173.938 \\
$\mathrm{E}_{i}=855.5 \mathrm{eV}$ & 234.074 & $\mathrm{~m}$ & $4 s{ }^{2} S_{1 / 2}-4 p{ }^{2} P_{1 / 2}$ & 233.77 & 2120 & 233.92 & 234.2 & 233.448 & 233.959 \\
\hline $\mathrm{Xe}^{24+} \mathrm{Zn}-\mathrm{like}$ & 164.352 & vs & $4 s^{2}{ }^{1} S_{0}-4 s 4 p{ }^{1} P_{1}$ & 162.98 & 2890 & 164.4 & 164.5 & & \\
$\mathrm{E}_{i}=817.8 \mathrm{eV}$ & 252.557 & $\mathrm{~m}$ & $4 s^{2}{ }^{1} S_{0}-4 s 4 p{ }^{3} P_{1}$ & 253.21 & 1380 & 252.44 & & & \\
\hline
\end{tabular}

\section{Line spectra and analysis}

Fig. 1 shows as an example a series of CCD images representing $\mathrm{Xe}^{17+}$ to $\mathrm{Xe}^{23+}$ lines observed in second order diffraction to resolve the details around $108 \AA$. Due to the close spacing of these lines, they could not be separated in first order. Two lines seen in the 608 to $708 \mathrm{eV}$ beam energy images are true first order lines at 195.598 $\AA$ for $\mathrm{Xe}^{21+}$ and at $196.795 \AA$ for $\mathrm{Xe}^{22+}$. For the determination of accurate line positions a horizontal, central 100 pixel wide strip was binned perpendicular to the direction of dispersion in order to circumvent the slight curvature of the slit image on the CCD blurring the wavelength information. Peaks in the binned spectra were fitted with Gaussian functions and the results are summarized in Table 1 and 2. The instrumental width is $0.23 \AA \mathrm{FWHM}$ and the total uncertainty amounts to $58 \mathrm{~m} \AA$ resulting from statistical error and uncertainty in the wavelength calibration.

\section{Comparison with calculations and pre- vious measurements}

To support the identification of the observed lines, wavelengths and intensities are calculated using the HULLAC computer package combined with a collisional-radiative model. For the $\mathrm{Cu}$ and Zn-like Xe ions a good agreement between our EBIT measurements, predictions by HULLAC and previous studies at the TFR [4], PLT $[3,10]$ and TEXT [11] tokamaks is found (Tab. 1). For lower charged Xe ions successively filling the $4 p$ subshell (Tab. 2) an increasing discrepancy between observed wavelengths and predicted values is noted. The values calculated with HULLAC are systematically too low, as well known, see Ref. [12]. Strong $4 s-4 p$ transitions dominate the line spectra of Xe but $4 p-4 d$ and $4 d-4 f$ transitions are also observed. Detailed calculations require an inclusion of many mixed configurations with electrons in all the subshells of $n=4$ and $n=5$. Most of the lines tabulated by Breton et al. [4] as originating from highly charged Xenon but not labelled with the emitting ion or the transition have now been identified with the EBIT technique and many could be classified with the corresponding transition.

\section{REFERENCES}

1. K.W. Hill, M.G. Bell, R.E. Bell, et al., Nucl. Fusion 39, 1949 (1999).

2. U. Stamm, H. Schwoerer, R. Lebert, Physik Journal 12, 33 (2002).

3. J.F. Seely, U. Feldman, A.W. Wouters, J.L. Schwob and S. Suckewer, Phys. Rev. A 40, 5020 (1989).

4. C. Breton, C. DeMichelis, W. Hecq, M. Mattioli, J. Ramette and B. Saoutic, C. BaucheArnoult, J. Bauche, J.F. Wyart, Physica Scripta 37, 33 (1988).

5. H.H. Hacker, R. Burhenn, K. Kondo, et al., Appl. Phys. B73, 59 (2001).

6. A. Bar-Shalom, M. Klapisch and J. Oreg, J. Quant. Spec. \& Rad. Transfer 71, 169 (2001).

7. C. Biedermann, A. Förster, G. Fußmann, R. Radtke, Physica Scripta T73, 360 (1997). 
Table 2

Experimental and theoretical wavelengths of Rb-like to Ga-like Xe ions in $[\AA]$. An estimate for the observed line intensity and a value for the predicted line intensity in [photons /s ion ] is included. For

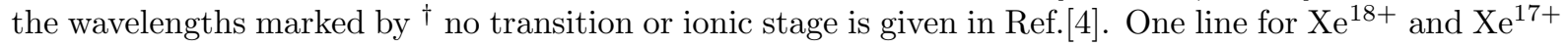
is not identified correctly by Breton et al. with respect to the charge state. The wavelength uncertainty amounts to $0.058 \AA$ for the BerlinEBIT experiment and $0.050 \AA$ for the TFR measurement.

\begin{tabular}{|c|c|c|c|c|c|c|}
\hline \multirow[t]{2}{*}{ Ion } & \multicolumn{2}{|c|}{ BerlinEBIT } & \multirow[t]{2}{*}{ Transition } & \multicolumn{2}{|c|}{ HULLAC } & \multirow{2}{*}{$\begin{array}{c}\text { TFR [4] } \\
\lambda\end{array}$} \\
\hline & $\lambda$ & Int. & & $\lambda$ & Int. & \\
\hline $\mathrm{Xe}^{23+}$ Ga-like & 113.59 & $\mathrm{~s}$ & $4 s^{2} 4 p_{1 / 2}[J=1 / 2]-4 s^{2} 4 d_{3 / 2}[J=3 / 2]$ & 111.27 & 2540 & $113.63^{\dagger}$ \\
\hline $\mathrm{E}_{i}=735.7 \mathrm{eV}$ & 160.503 & $\mathrm{~m}$ & $4 s^{2} 4 p_{3 / 2}[3 / 2]-4 s\left[4 p_{3 / 2}^{2}(2)\right][3 / 2]$ & 153.76 & 969 & $160.58^{\dagger}$ \\
\hline \multirow{3}{*}{$\begin{array}{l}\text { g.s. } 4 s^{2} 4 p_{1 / 2} \\
{[J=1 / 2]}\end{array}$} & 162.470 & $\mathrm{~s}$ & $4 s^{2} 4 p_{1 / 2}[1 / 2]-\left[4 s 4 p_{1 / 2}(1)\right] 4 p_{3 / 2}[1 / 2]$ & 157.22 & 2890 & $162.52^{\dagger}$ \\
\hline & 171.092 & vs & $4 s^{2} 4 p_{1 / 2}[1 / 2]-\left[4 s 4 p_{1 / 2}(1)\right] 4 p_{3 / 2}[3 / 2]$ & 168.00 & 2690 & $171.60^{\dagger}$ \\
\hline & 192.084 & vw & $4 s^{2} 4 p_{3 / 2}[3 / 2]-4 s\left[4 p_{3 / 2}^{2}(2)\right][5 / 2]$ & 190.89 & 198 & $192.40^{\dagger}$ \\
\hline $\mathrm{Xe}^{22+}$ Ge-like & 112.38 & $\mathrm{~s}$ & $4 s^{2} 4 p_{1 / 2}^{2}[0]-4 s^{2} 4 p_{1 / 2} 4 d_{3 / 2}[1]$ & 109.66 & 4710 & $112.40^{\dagger}$ \\
\hline \multirow{7}{*}{$\begin{array}{l}\mathrm{E}_{i}=699.8 \mathrm{eV} \\
\text { g.s. } 4 s^{2} 4 p_{1 / 2}^{2} \\
{[J=0]}\end{array}$} & 114.32 & vw & $4 s^{2} 4 p_{1 / 2} 4 p_{3 / 2}[1]-4 s^{2} 4 p_{3 / 2} 4 d_{3 / 2}[2]$ & 110.90 & 621 & \\
\hline & 114.725 & $\mathrm{w}$ & $4 s^{2} 4 p_{1 / 2} 4 p_{3 / 2}[2]-4 s^{2} 4 p_{3 / 2} 4 d_{3 / 2}[3]$ & 111.54 & 2450 & 114.84 \\
\hline & 159.93 & $\mathrm{w}$ & $4 s^{2} 4 p_{1 / 2} 4 p_{3 / 2}[2]-4 s 4 p_{1 / 2}(1) 4 p_{3 / 2}^{2}[1]$ & 151.34 & 1580 & $159.93^{\dagger}$ \\
\hline & 171.42 & $\mathrm{w}$ & $4 s^{2} 4 p_{1 / 2} 4 p_{3 / 2}[2]-4 s 4 p_{1 / 2}(1) 4 p_{3 / 2}^{2}[2]$ & 166.73 & 1080 & \\
\hline & 173.003 & $\mathrm{~m}$ & $4 s^{2} 4 p_{1 / 2}^{2}[0]-4 s 4 p_{1 / 2}^{2} 4 p_{3 / 2}[1]$ & 168.92 & 2820 & $173.23^{\dagger}$ \\
\hline & 191.461 & vw & $4 s^{2} 4 p_{1 / 2} 4 p_{3 / 2}[2]-4 s 4 p_{1 / 2}(1) 4 p_{3 / 2}^{2}[3]$ & 188.33 & 507 & \\
\hline & 196.795 & vw & $4 s^{2} 4 p_{1 / 2} 4 p_{3 / 2}[2]-4 s 4 p_{1 / 2}(0) 4 p_{3 / 2}^{2}[2]$ & 193.93 & 425 & \\
\hline \multirow{9}{*}{$\begin{array}{l}\mathrm{Xe}^{21+} \text { As-like } \\
\mathrm{E}_{i}=650.3 \mathrm{eV} \\
4 s^{2} 4 p_{1 / 2}^{2} 4 p \\
{[J=3 / 2]}\end{array}$} & 111.715 & $\mathrm{~m}$ & $4 s^{2} 4 p_{1 / 2}^{2} 4 p_{3 / 2}[3 / 2]-4 s^{2} 4 p_{1 / 2} 4 p_{3 / 2}(2) 4 d_{3 / 2}[1 / 2]$ & 107.41 & 1430 & $111.69^{\dagger}$ \\
\hline & & & $4 s^{2} 4 p_{1 / 2}^{2} 4 p_{3 / 2}[3 / 2]-4 s^{2} 4 p_{1 / 2} 4 p_{3 / 2}(2) 4 d_{3 / 2}[3 / 2]$ & 107.95 & 1350 & \\
\hline & 112.775 & $\mathrm{~m}$ & $4 s^{2} 4 p_{1 / 2}^{2} 4 p_{3 / 2}[3 / 2]-4 s^{2} 4 p_{1 / 2} 4 p_{3 / 2}(1) 4 d_{3 / 2}[5 / 2]$ & 108.64 & 2480 & $112.80^{\dagger}$ \\
\hline & 116.33 & vw & $4 s^{2} 4 p_{1 / 2}^{2} 4 p_{3 / 2}[3 / 2]-4 s^{2} 4 p_{1 / 2} 4 p_{3 / 2}(1) 4 d_{5 / 2}[3 / 2]$ & 111.61 & 562 & $116.45^{\dagger}$ \\
\hline & 166.67 & vw & $4 s^{2} 4 p_{1 / 2} 4 p_{3 / 2}^{2}(2)[5 / 2]-4 s 4 p_{1 / 2}(1) 4 p_{3 / 2}^{3}[3 / 2]$ & 160.36 & 999 & $166.30^{\dagger}$ \\
\hline & 173.805 & $\mathrm{~m}$ & $4 s^{2} 4 p_{1 / 2} 4 p_{3 / 2}^{2}(2)[3 / 2]-4 s 4 p_{1 / 2}^{2} 4 p_{3 / 2}^{2}(0)[1 / 2]$ & 168.56 & 1110 & $173.91^{\dagger}$ \\
\hline & 175.055 & $\mathrm{~m}$ & $4 s^{2} 4 p_{1 / 2}^{2} 4 p_{3 / 2}[3 / 2]-4 s 4 p_{1 / 2}^{2} 4 p_{3 / 2}^{2}(2)[3 / 2]$ & 170.30 & 1470 & \\
\hline & 187.11 & $\mathrm{vw}$ & $4 s^{2} 4 p_{1 / 2} 4 p_{3 / 2}^{2}(2)[3 / 2]-4 s 4 p_{1 / 2}(0) 4 p_{3 / 2}^{3}[3 / 2]$ & 179.91 & 1340 & \\
\hline & 195.598 & $\mathrm{w}$ & $4 s^{2} 4 p_{1 / 2}^{2} 4 p_{3 / 2}[3 / 2]-4 s 4 p_{1 / 2}^{2} 4 p_{3 / 2}^{2}(2)[5 / 2]$ & 191.27 & 924 & \\
\hline \multirow{4}{*}{$\begin{array}{l}\mathrm{Xe}^{20+} \text { Se-like } \\
\mathrm{E}_{i}=615.9 \mathrm{eV} \\
4 s^{2} 4 p_{1 / 2}^{2} 4 p_{3 / 2}^{2} \\
{[J=2]}\end{array}$} & 108.565 & $\mathrm{~m}$ & $4 s^{2} 4 p_{1 / 2}^{2} 4 p_{3 / 2}^{2}[2]-4 s^{2}\left[4 p_{1 / 2} 4 p_{3 / 2}^{2}(2)\right](3 / 2) 4 d_{3 / 2}[3]$ & 103.62 & 3760 & $108.35^{\dagger}$ \\
\hline & 110.60 & $\mathrm{w}$ & $4 s^{2} 4 p_{1 / 2}^{2} 4 p_{3 / 2}^{2}[2]-4 s^{2}\left[4 p_{1 / 2} 4 p_{3 / 2}^{2}(2)\right](5 / 2) 4 d_{3 / 2}[1]$ & 105.15 & 1410 & \\
\hline & 110.93 & $\mathrm{~m}$ & $4 s^{2} 4 p_{1 / 2}^{2} 4 p_{3 / 2}^{2}[2]-4 s^{2}\left[4 p_{1 / 2} 4 p_{3 / 2}^{2}(2)\right](5 / 2) 4 d_{3 / 2}[2]$ & 105.56 & 1870 & $110.84^{\dagger}$ \\
\hline & 111.495 & $\mathrm{~m}$ & $4 s^{2} 4 p_{1 / 2}^{2} 4 p_{3 / 2}^{2}[2]-4 s^{2}\left[4 p_{1 / 2} 4 p_{3 / 2}^{2}(2)\right](5 / 2) 4 d_{5 / 2}[3]$ & 107.17 & 1010 & \\
\hline \multirow{4}{*}{$\begin{array}{l}\mathrm{Xe}^{19+} \text { Br-like } \\
\mathrm{E}_{i}=582.4 \mathrm{eV} \\
4 s^{2} 4 p_{1 / 2}^{2} 4 p_{3 / 2}^{3} \\
{[J=3 / 2]}\end{array}$} & 108.845 & vs & $4 s^{2} 4 p_{3 / 2}^{5}[3 / 2]-4 s^{2}\left[4 p_{3 / 2}^{3} 4 p_{1 / 2}\right](1) 4 d_{3 / 2}[5 / 2]$ & 102.39 & 5700 & $108.84^{\dagger}$ \\
\hline & & & $4 s^{2} 4 p_{3 / 2}^{5}[3 / 2]-4 s^{2}\left[4 p_{3 / 2}^{3} 4 p_{1 / 2}\right](2) 4 d_{3 / 2}[3 / 2]$ & 102.29 & 2530 & \\
\hline & 109.450 & $\mathrm{~m}$ & $4 s^{2} 4 p_{3 / 2}^{5}[3 / 2]-4 s^{2}\left[4 p_{3 / 2}^{3} 4 p_{1 / 2}\right](1) 4 d_{3 / 2}[3 / 2]$ & 104.38 & 1880 & $109.50^{\dagger}$ \\
\hline & 111.895 & $\mathrm{~m}$ & $4 s^{2} 4 p_{3 / 2}^{5}[3 / 2]-4 s^{2}\left[4 p_{3 / 2}^{3} 4 p_{1 / 2}\right](1) 4 d_{5 / 2}[3 / 2]$ & 107.34 & 707 & $111.92^{\dagger}$ \\
\hline \multirow{2}{*}{$\begin{array}{l}\mathrm{Xe}^{18+} \text { Kr-like } \\
\mathrm{E}_{i}=549.1 \mathrm{eV} \\
4 s^{2} 4 p^{6}[J=0]\end{array}$} & 108.35 & vs & $4 s^{2} 4 p^{6}[0]-4 s^{2} 4 p_{1 / 2}^{5} 4 d_{3 / 2}[1]$ & 101.09 & 12700 & 108.35 \\
\hline & & & & & & $(19+)$ \\
\hline \multirow{2}{*}{$\begin{array}{l}\mathrm{Xe}^{17+} \text { Rb-like } \\
\mathrm{E}_{i}=434.2 \mathrm{eV}\end{array}$} & 106.285 & $\mathrm{w}$ & $4 p^{6} 4 d_{3 / 2}[3 / 2]-4 p_{1 / 2}^{5} 4 d_{3 / 2}^{2}[3 / 2]$ & 101.16 & 3680 & 106.37 \\
\hline & & & $4 p^{6} 4 d_{3 / 2}[3 / 2]-4 p_{1 / 2}^{5} 4 d_{3 / 2}(1) 4 d_{5 / 2}[3 / 2]$ & 101.64 & 2520 & $(18+)$ \\
\hline \multirow{3}{*}{$\begin{array}{l}4 s^{2} 4 p^{6} 4 d_{3 / 2} \\
{[J=3 / 2]}\end{array}$} & 107.185 & $\mathrm{~m}$ & $4 p^{6} 4 d_{5 / 2}[5 / 2]-4 p_{1 / 2}^{5} 4 d_{3 / 2}(1) 4 d_{5 / 2}[7 / 2]$ & 102.54 & 10500 & $107.24^{\dagger}$ \\
\hline & 107.92 & $\mathrm{~m}$ & $4 p^{6} 4 d_{3 / 2}[3 / 2]-4 p_{1 / 2}^{5} 4 d_{3 / 2}(1) 4 d_{5 / 2}[5 / 2]$ & 102.88 & 9290 & $107.94^{\dagger}$ \\
\hline & 109.45 & $\mathrm{w}$ & $4 p^{6} 4 d_{3 / 2}[3 / 2]-4 p^{6} 4 f_{5 / 2}$ & 104.75 & 7940 & $109.50^{\dagger}$ \\
\hline
\end{tabular}


8. C. Biedermann, R. Radtke J.L. Schwob, P. Mandelbaum, R. Doron, T. Fuchs, G. Fußmann, Physica Scripta T92, 85 (2001).

9. R.L. Kelly, J. Phys. Chem. Ref. Data 16 (1987), available online at http://cfawww.harvard.edu/amdata/ampdata/kelly/kelly.html.

10. E. Hinnov, Phys. Rev. A14, 1533 (1976).

11. V. Kaufman, J. Sugar, W.L. Rowan, J. Opt. Soc. Am. 5, 1273 (1988).

12. R. Radtke, C. Biedermann, J.L. Schwob, P. Mandelbaum, R. Doron, Phys. Rev. A64, 012720 (2001). 